\title{
Prevalencia de úlceras por presión en pacientes adultos hospitalizados
}

González-Flores Silvia Patricia*, Jiménez-Camarena Rosalba*, Rodríguez-Alvarado Martha*, Rodríguez-García Lourdes Adriana*, Silva-Villalobos Juana María*, Gómez-Cardona Juan Pablo**, Rodríguez Nava Víctor Federico**, Rodríguez Ramírez José Manuel**.

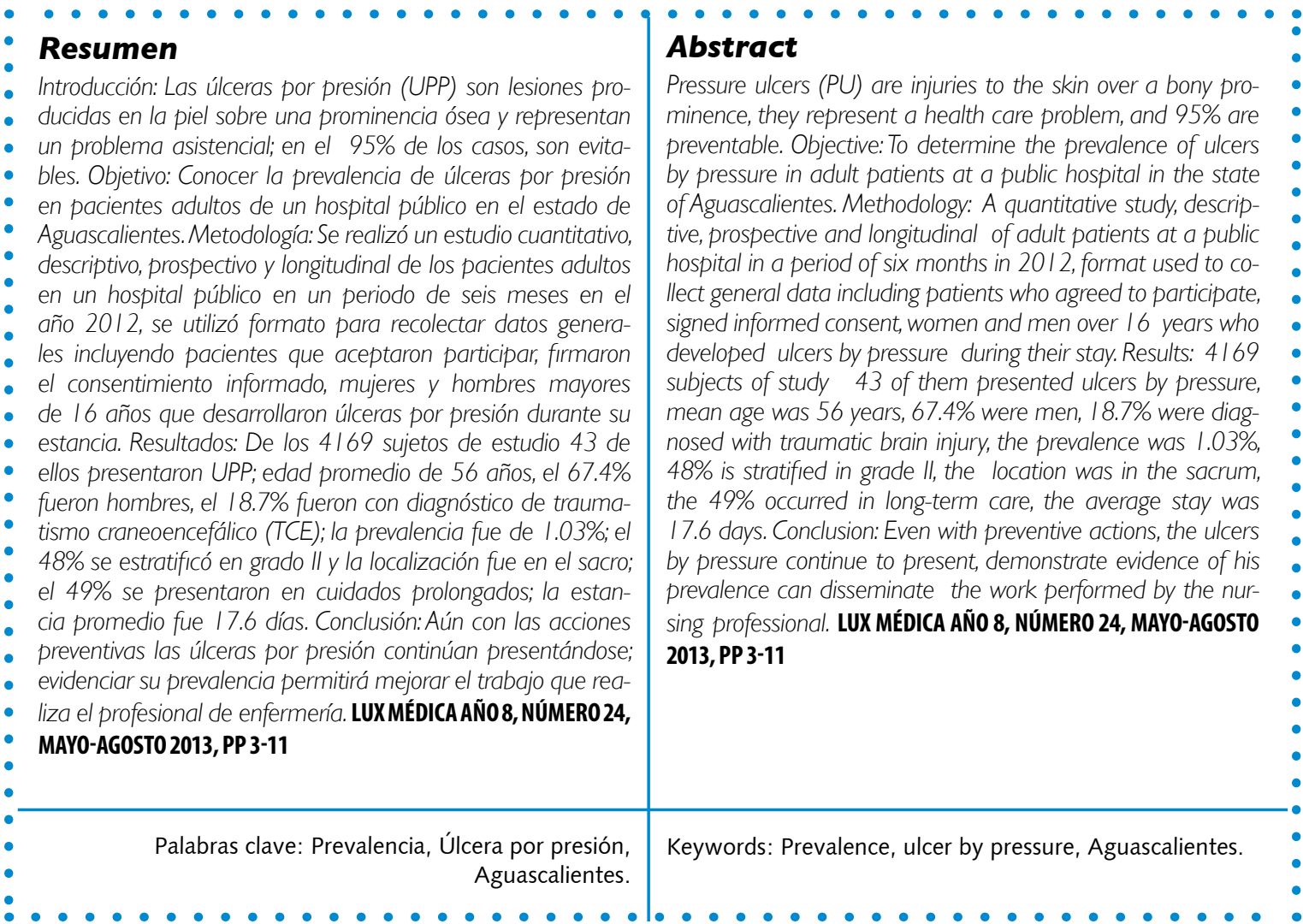

* Licenciadas en Enfermería de la Universidad Autónoma de Aguascalientes.

** Profesores de tiempo completo, investigadores del Departamento de Enfermería de la UAA

Fecha de recibido: 18 de abril 2013

Fecha de aceptación: 26 de julio de 2013

Correspondencia: Silvia Patricia González Flores. Departamento de Enfermería del Centro de Ciencias de la Salud de la Universidad Autónoma de Aguascalientes. Av. Universidad No 940 Cd. Universitaria. Edificio 105. CP 20131. Aguascalientes, Ags., México. Tel. 9108437. E-mail: spgonza@ correo.uaamx 


\section{Introducción}

Las úlceras por presión (UPP), conocidas popularmente como llagas, escaras ó úlceras por decúbito, son un problema común que se presenta en pacientes hospitalizados ó no, que permanecen inmovilizados por periodos prolongados. Se presentan con mayor frecuencia en pacientes de edad avanzada. Son el resultado de la destrucción cutánea, de la necrosis del tejido subyacente, grasa y músculo, producido por una restricción de flujo sanguíneo, principalmente por una prominencia ósea ${ }^{1}$. Influyen también factores intrínsecos y extrínsecos como la edad, tiempo de hospitalización e infección, uso de sedación, concentraciones bajas de albúmina, la humedad facilitada por la sudoración y la incontinencia fecal2; el principal mecanismo de lesión es el aplastamiento de los tejidos entre dos planos el correspondiente del paciente y otro externo a él. ${ }^{3-5}$ La trascendencia como problema de salud, abarca el ámbito mundial y repercute en lo personal y familiar, además del gran costo económico que representan para los sistemas de salud, debido a que estos pacientes requieren gran cantidad de recursos médicos (humanos y materiales) a su disposición y permanecen hospitalizados por largos períodos, lo cual hace que aumenten considerablemente los gastos asistenciales ${ }^{6}$; además es un indicador de calidad en la esencia del trabajo de enfermería. Las UPP se clasifican en cuatro estadios s, $^{3,7,8}$ (tabla 1), en una persona con pigmentación oscura de la piel, el estadio I puede aparecer como una decoloración persistente roja, azul, o púrpura. La etapa de una úlcera no puede ser determinada hasta que el tejido necrótico o costra se retira para exponer la base de la herida.

\section{Tabla}

Sistema de estadificación para las úlceras por presión

\begin{tabular}{|l|l|}
\hline Estadio & Descripción \\
\hline I & $\begin{array}{l}\text { Eritema cutáneo que no palidece en piel intacta, generalmente sobre una prominencia } \\
\text { ósea. La piel pigmentada oscura no puede tener palidez visible, y el área afectada pueden } \\
\text { diferir de los alrededores, el tejido afectado puede ser doloroso, caliente o en comparación } \\
\text { con el tejido adyacente. }\end{array}$ \\
\hline II & $\begin{array}{l}\text { Pérdida parcial del grosor de la piel, que puede afectar la epidermis y/o dermis; con } \\
\text { aspecto de abrasión o ampolla. }\end{array}$ \\
\hline III & Pérdida total del grosor de la piel que implica lesión o necrosis del tejido subcutáneo que \\
& puede extenderse hacia abajo, pero no a la fascia subyacente. \\
\hline IV & Pérdida total del grosor de la piel con destrucción masiva, necrosis tisular o lesión en el \\
& músculo, hueso o estructura de sostén. \\
\hline
\end{tabular}

Fuente: National Pressure Ulcer Advisory Panel (www.npuap.org)

El $95 \%$ de las UPP son prevenibles ${ }^{9}$, son un proceso banal, fatal, inevitable y silente ${ }^{10}$; es un reto al que se enfrentan los profesionales de salud en su práctica asistencial siendo la prevención el primer paso para evitarlas. En España, desde 1999 el Grupo Nacional para el Estudio y Asesoramiento en Úlceras por presión y Heridas Crónicas (GNEAUPP) es un conjunto de profesionales que se encuentra trabajando 
junto con otros organismos internacionales, como la Asociación Europea para el manejo de las heridas o la estadounidense National Pressure Ulcer Advisory Panel.

Se calcula que su incidencia en la población general es del 1,7\% entre los 55 y 69 años y del 3,3\% entre los 70 y 75 años $^{11}$. La prevalencia reportada a nivel mundial de acuerdo a la Organización Mundial de la Salud (OMS) está entre el 5 y 12\%, en América es del $7 \%$ y en México en las instituciones de salud oscila entre el 4,5 y $13 \%^{12}$. La prevalencia más alta de las UPP, se observa en adultos mayores, según los estudios revisados de diferentes países: España ${ }^{13}$, Brasil, Estados Unidos, México y Chile.

Arango y colaboradores, en su Tratado de Geriatría, acotan los siguientes puntos acerca de la incidencia y prevalencia de las úlceras por presión; basados en las estadísticas que el Grupo Nacional para el Estudio y Asesoramiento en Úlceras por Presión y Heridas Crónicas de España que se publica anualmente ${ }^{14}$ :

- Entre un 3 - 11\% de los pacientes que ingresan en los hospitales desarrollan UPP.

- Cerca del 70\% de las úlceras por presión se producen en las primeras dos semanas de hospitalización.

- La guía de Intervención de enfermería basada en la evidencia científica creada en el 2005 por la Asociación Colombiana de Facultades de Enfermería (ACOFAEN), en su apartado de "úlceras", presenta los siguientes datos epidemiológicos ${ }^{15}$ :

- 3 a $11 \%$ de los pacientes hospitalizados, presentan úlceras por presión según estudios realizados en Europa, Sur África y Canadá.

- En Estados Unidos, la prevalencia de las UPP en hospitales de tercer nivel es de $8,5 \%$, en centros para veteranos es de $7,4 \%$ y en casas de enfermería es de $29,3 \%$; la prevalencia de las personas con UPP en el centro hospitalario pue- den llegar hasta el $45 \%$ en los enfermos crónicos y por arriba del $9 \%$ de todas las personas hospitalizadas, constituyéndose en un problema de salud, no solo en personas en estado crítico sino también en personas en estado agudo.

- La incidencia de personas con UPP en hospitales americanos se encuentra en un rango de 2,7 y un $29,5 \%$.

- En pacientes críticos, la incidencia de las UPP suele constituir una importante complicación. En Estados Unidos, en el año de 1987, estas lesiones se presentaron en el $40 \%$ de pacientes de la unidad de cuidados intensivos (UCI). En el 2001, la cifra anterior se redujo a un rango entre 3,8 y el $8 \%$.

- En España en 1995 se publicó una incidencia del $21 \%$ en pacientes de cuidados intensivos, del $17 \%$ en el año 1997 y del $6,4 \%$ en el año 2000 , aunque recientemente han descrito una incidencia tan elevada como del $22,8 \%$ en una $\mathrm{UCl}$.

Según Flemming K, (2008), en el Reino Unido, las úlceras por presión se registraron de un $5 \%$ a $32 \%$ de pacientes ingresados en un hospital general de distrito y en un $4 \%$ al $7 \%$ de pacientes en ámbitos comunitarios $^{16}$.

Estudios nacionales como el realizado en el Instituto Nacional de Enfermedades Respiratorias (INER) en el año 2004 registró una prevalencia del $80 \%$, siendo los talones el área más afectada con un $29 \%$ siguiéndole el sacro, maléolos y codos; el grupo de mayor riesgo son los pacientes sometidos a ventilación mecánica en la terapia intensiva con un $86 \%{ }^{1}$.

El Instituto Nacional de Cardiología Ignacio Chávez (INCICh) México, realizó un estudio en el año 2007-2008 donde la prevalencia de UPP fue de $1,5 \%$, el $58 \%$ se presentó en el género masculino, se presentaron en pacientes mayores de 60 años con el $47,3 \%$, con respecto a la estratificación de la úlcera el 58,3\% fue grado II, en la unidad de cuidados intensivos ${ }^{17}$. 
México ${ }^{18}$, en su primer estudio de prevalencia a nivel nacional publicado en el 2013 reportó que las entidades federativas con mayor prevalencia cruda de UPP fueron Jalisco $(64,08 \%)$, Querétaro $(41,86 \%)$, Campeche $(38,15 \%)$ y Colima $(33,33 \%)$; así mismo, las entidades federativas con mayor prevalencia media fueron Veracruz $(72,96 \%)$, Campeche $(60 \%)$, Colima $(50,7 \%)$, Jalisco $(46,12 \%)$ y Baja California $(45,8 \%)$. Las mujeres tuvieron una mayor prevalencia de UPP (52\%) y los pacientes que tienen entre 71 y 80 años (20\%).

En cuanto a los días de aparición de las UPP, el promedio fue de seis días después de la fecha de ingreso; $26 \%$ de los pacientes llegaron a la unidad médica con UPP desarrolladas y $74 \%$ desarrollaron UPP después de su ingreso a la unidad médica.

Con relación al sitio anatómico, se encontró que la frecuencia de la aparición fue: sacro $27 \%$, talón $27 \%$, isquion $10 \%$, cabeza $4 \%$ y codos $3 \%$. La severidad de las UPP de acuerdo con diversos estadios fue: estadio I 39\%, estadio II 34\%, estadio III 12\%, estadio IV 5\%, sin estadio 10\%. Por lo que a nivel nacional, el porcentaje de prevalencia cruda fue de $12,92 \%$, en tanto que la prevalencia media fue de $20,0 \pm 15,7 \%$ : E objetivo de este estudio fue conocer la prevalencia de úlceras por presión en pacientes adultos hospitalizados en un hospital público del municipio de Aguascalientes, Ags., México.

\section{| | | | | | | | | | | | | | | | | | | | | | | | | | | | | | | | | | | | | | | | | | | | | | | | | | | | | | | | | | | | | | | | | | | | | | | | | | | | | | | | | | | | | | | | | | | | | | | | | | | | | | | ||}

\section{Material y métodos}

Se realizó un estudio descriptivo, prospectivo y transversal en los servicios: cuidados prolongados, urgencias hospitalización, unidad de cuidados intensivos general, intermedios y unidad de cuidados coronarios, cirugía mujeres y cirugía hombres en un hospital público del municipio de Aguascalientes. En el periodo del 1 de abril al 30 de septiembre del 2012; el muestreo fue por conveniencia. La recolección de datos se obtuvo mediante la revisión de los registros de ingreso de los pacientes adultos, identificación del paciente, presentación ante el paciente o familiar, explicación del motivo de la investigación. Se valoró al paciente para detectar el riesgo de presentar UPP al ingreso y durante su estancia hospitalario registrándolas variables encontradas posteriores a la valoración y monitorización de la lesiones en el formato diseñado (Anexo I). Se elaboró una base de datos con las variables: nombre del servicio, fecha de ingreso, edad, género, diagnóstico, estratificación, localización anatómica de la UPP en el paquete estadístico SPSS versión 19.0. Se analizaron los datos a través de la estadística descriptiva: medias, frecuencias, porcentajes, mínimos y máximos.

| | | | | | | | | | | | | | | | | | | | | | | | | | | | | | | | | | | | | | | | | | | | | | | | | | | | | | | | | | | | | | | | | | | | | | | | | | | | | | | | | | | | | | | | | | | | | | | | | | | | | | | | | | | | | | | | | |

\section{Resultados}

4,169 pacientes adultos ingresaron durante el periodo comprendido de la investigación (tabla 2), 43 presentaron UPP, por lo que la prevalencia en el periodo estudia- do fue de $1,03 \%$, de los cuales el $67,4 \%$ fueron pacientes del género masculino y el $32,6 \%$ género femenino. 


\section{Tabla 2}

Frecuencia de UPP en pacientes según el servicio de hospitalización

\begin{tabular}{|lcc|}
\hline \multicolumn{1}{c}{ Servicio } & Número de Ingresos & UPP \\
\hline Urgencias & 2046 & 0 \\
\hline UCIG & 117 & 16 \\
\hline UTIN & 94 & 2 \\
\hline ICIC & 99 & 3 \\
\hline Cuidados prolongados & 104 & 21 \\
\hline Cirugía mujeres & 828 & 0 \\
\hline Cirugía hombres & 881 & 1 \\
\hline Total & 4169 & 43 \\
\hline
\end{tabular}

Fuente: Hospital público. Abril-Septiembre 2012

La edad promedio de los pacientes 47,8\% fue grado II (gráfica1) y el sitio de que presentaron UPP fue de 56 años. De localización más frecuente fue en el sacro acuerdo a la estratificación de la úlcera el con un $44 \%$ (gráfica 2).

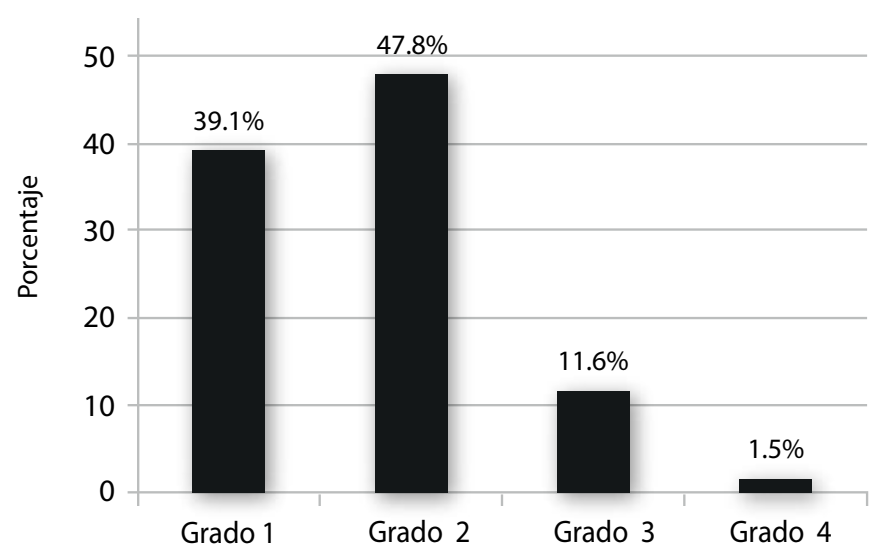

Gráfica 1. Distribución por estadio de severidad en los pacientes con úlceras por presión.

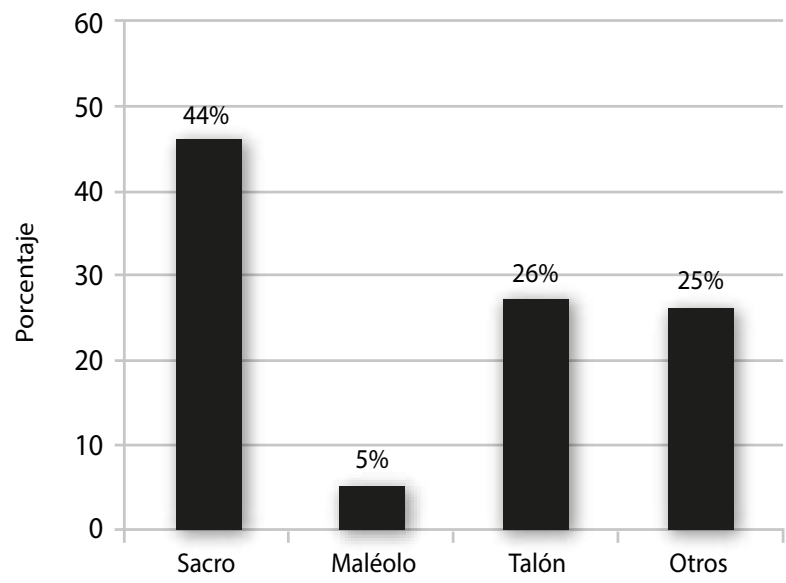

Gráfica 2. Distribución de los pacientes con úlceras por presión según su localización. 
En cuanto al diagnóstico los pacientes con traumatismo craneoencefálico/policontundido representan el $18,7 \%$ ya que por su estado hemodinámico inestable influye en la movilización y/o cambios posturales (gráfica 3). Dentro del 39,5\% se encuentran los diagnósticos de menor prevalencia como es la artritis séptica, choque séptico, clipaje de aneurisma, crisis tiroidea, disección aórtica, fractura de cadera, hemorragia parenquimatosa, entre otros.

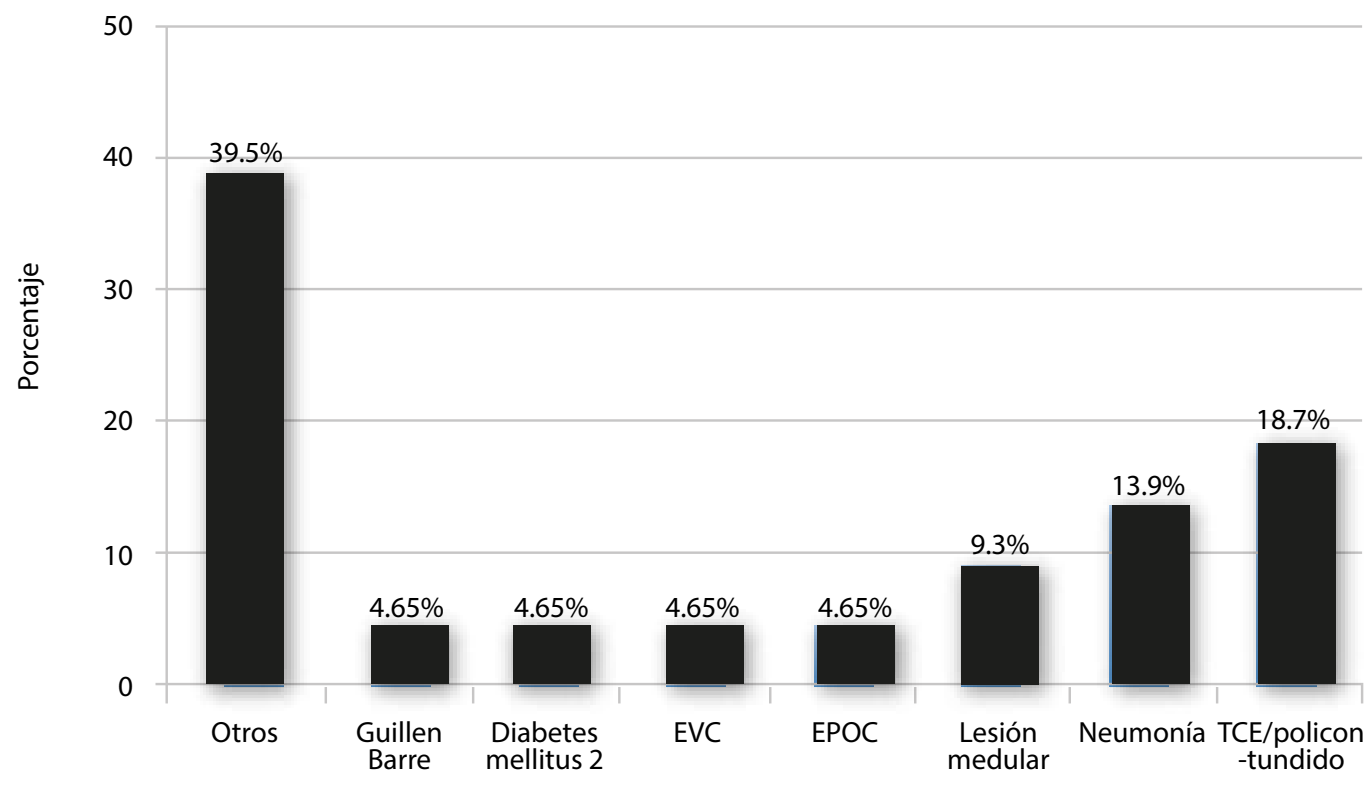

Gráfica 3. Distribución de las UPP según el diagnóstico de ingreso Fuente: Hospital público. AbrilSeptiembre 2012

Por último el promedio de los días estancia hospitalaria de los pacientes con UPP fue de 17,6+14 indicándonos que los pacientes de este hospital de segundo nivel, tienen un rango amplio de permanencia hospitalaria de 4 hasta 31 días.

\section{| | | | | | | | | | | | | | | | | | | | | | | | | | | | | | | | | | | | | | | | | | | | | | | | | | | | | | | | | | | | | | | | | | | | | | | | | | | | | | | | | | | | | | | | | | | | || ||}

\section{Discusión}

Es evidente que aún sigue siendo un problema de salud pública las UPP, aun cuando se han establecidos protocolos de prevención, cuidado y tratamiento para las mismas. Sin duda alguna la difusión de los datos epidemiológicos nos hacen ver el impacto ante la sociedad, además permite la implementación y estandarización de nuevos protocolos de prevención y manejo de las UPP en los tres niveles de atención médica, pues las UPP prevalecen en ellos como lo reporta un estudio de prevalencia de UPP en la Comunidad Autónoma de la Rioja en 1999, en donde la prevalencia de UPP en hospitales agudos fue de $12.26 \%$, en las instituciones socio sanitarias de $12,84 \%$ y en la comunidad de $0,26 \%{ }^{19}$.

Es de suma importancia los cuidados preventivos para evitar estos eventos adversos, la persona enferma que se encuentra en una cama debe ser movilizado, mientras que en una institución hospitalaria, el personal de enfermería es quien realiza esta intervención para de alguna manera evitar la restricción del flujo san- 
guíneo como lo menciona Bautista et al. $2004^{1}$, y por lo tanto una destrucción cutánea y/o tisular.

La prevalencia de UPP, obtenida en este estudio fue relativamente pequeña, por debajo de la prevalencia a nivel mundial reportada por la OMS, ACOFAEN, Reino Unido, INER (Instituto Nacional de Enfermedades Respiratorias, coincide con el estudio realizado en el Instituto Nacional de Cardiología Ignacio Chávez (INCICh) México en el año 2007-2008 donde la prevalencia fue de $1,5 \%$ presentándose más en el género masculino (58\%), mayores de 60 años (47,3\%), el estadio II (58,3\%) y en la unidad de cuidados intensivos ${ }^{10}$, en nuestro estudio fue de $1,03 \%$, el $67,4 \%$ las presentó el género masculino, el $48 \%$ en estadio II y el $49 \%$ en el servicio de cuidados prolongados y el $37 \%$ en la unidad de cuidados intensivos .

Resulta interesante el incremento de las UPP en el adulto mayor, en la unidad de cuidados intensivos, si bien sabemos este tipo de unidades requiere de cuidados especializados por el tipo de pacientes que ingresa, son pacientes que en su mayoría cursan con ayunos prolongados ocasionándoles un déficit nutricional, tratamiento farmacológico amplio, un estado hemodinámico inestable impidiendo que se rea- licen cambios posturales y por lo tanto la movilización, dando así inicio a este problema de salud pública que compete principalmente al profesional de enfermería.

Los datos anteriores nos conduce a implementar medidas sanitarias para su atención, teniendo en cuenta que son múltiples los factores que nos conllevan a la aparición de una UPP, como lo es el mismo diagnóstico del paciente, cursar con una enfermedad aguda y o crónica, su tratamiento farmacológico, estado nutricional siendo este un factor muy importante, la estancia hospitalaria, o el simple hecho de cómo se moviliza a la persona en su cama pues es notorio que al desplazarlo hay fricción.

La disminución de la prevalencia de las UPP implica conocer datos epidemiológicos, implementación de protocolos para la prevención oportuna y sobre todo interés de brindar cuidados con calidad a nuestros pacientes tomando en cuenta cada una de las características de los pacientes que desarrollan UPP, pues además de ser evitables como lo menciona la enfermera británica $P$. Hibbs también son una negligencia profesional con importantes implicaciones legales y éticas para los profesionales asistenciales, así como para las instituciones en la que éstos prestan sus servicios y los gestores de la misma ${ }^{20}$.

\section{Conclusiones}

La prevalencia de UPP en este hospital de segundo nivel es baja, sin embargo sigue siendo un problema de salud pública por la repercusión en lo personal, social y económico. Si bien sabemos, las UPP son un indicador de calidad en la asistencia de enfermería, por lo que es importante tomar medidas preventivas desde el momento que ingrese el paciente hasta su egreso.

Es necesario que las instituciones hospitalarias reporten este evento adverso para conocer el impacto ante la sociedad sanitaria para el establecimiento de protocolos de prevención y tratamiento, para mejorar la calidad de los servicios de salud; que día con día se le valore al paciente el riesgo de desarrollar una UPP a través de la escala de Braden, educar a aquellos pacientes que estén en condiciones de moviliza- 
ción la importancia de una movilización continua involucrando al familiar, hacer uso de colchones alternos, proteger prominencias óseas, vigilar y cuidar la piel (inspeccionar la presencia de eritema o alguna otra lesión en la piel, mantener limpia y seca, lubricarla con cremas humectantes), evitar la fricción en el paciente al momento de bañarlo, cambiar la ropa de su cama o movilizarlo, también es muy importante el estado nutricional por lo que se sugiere evitar el ayuno prolongado y de lo contrario sugerir la indicación de un aporte nutricio de manera temprana en su manejo médico.

\section{Bibliografía}

1. Bautista P L, Esparza GM.M., Ortega AJ., Las úlceras por presión en pacientes sometidos a ventilación mecánica en la Unidad de cuidados intensivos e intermedios del INER. Rev. Inst. Nal. Enf. Resp. Mex. 2004; 17(2): 91-99.

2. Yepes D, Molina F, León W, Pérez E. Incidencia y factores de riesgo en relación con las úlceras por presión en enfermos críticos. Med Intensiva.2009; 33(06):276-81.

3. L. J. Cowan, J. K. Stechmiller, M. Rowe et al., "Enhancing Braden pressure ulcer risk assessment in acutely ill adult veterans," Wound Repair and Regeneration. 2012; 20:137-148.

4. Pressure Ulcer Stages Revised by NPUAP, National Pressure Ulcer Advisory Panel, Press Release, Washington, DC, USA, 2007.

5. J. Kottner, K. Raeder, R. Halfens, and T. Dassen, "A systematic review of interrater reliability of pressure ulcer classification systems," Journal of Clinical Nursing. 2009; 18(3): 315-336.

6. Alfonso Coto JC, Cairos Báez J, López Cabrera LD. Cirugía reconstructiva de las úlceras por presión. Rev Cubana Cir. 2007; 46(3).

7. National Pressure Ulcer Advisory Panel. Updated staging system. http://www.npuap.org/pr2.htm.

8. Clasificación-Estadiaje de las úlceras por presión. Grupo Nacional para el Estudio y Asesoramiento en Úlceras por Presión y Heridas Crónicas (GNEAUPP). Doc. II. Disponible en: www.gneaupp.org.

9. Hibbs, P. Pressure area care for the city \& Hackney Health Athority; London: St. Bartholomews Hospital: 1987.

10. Soldevilla Agreda JJ. Las úlceras por presión en Gerontología. Dimensión epidemiológica, económica, ética y legal. Tesis Doctoral. Santiago de Compostela: Universidad de Santiago, 2007.

11. Riesgo de aparición de ulceras por presión. Disponible en: http://www.enfermero.cl/index. php?option $=$ com_content $\&$ view $=$ article $\&$ id $=752: r$ iesgo-de-aparicion-de-ulceras-por-presion \&catid $=3$ 8: publicaciones\&ltemid $=235$.
12. Cantún UF, Uc ChN. Perfil de riesgo para el desarrollo de úlceras por presión en pacientes adultos hospitalizados. Rev Enferm IMSS 2005; 13(3): 147-152.

13. Díaz Pizarro J.M., García Sánchez A.S., Núñez Ballesteros, A., Osorio Díaz, R. Prevalencia de las úlceras por presión en una residencia asistida de mayores. Gerokomos.2007; 18(3), 52-55.

14. ARANGO Claudia. ÚLCERAS por Presión, en: I Tratado de Geriatría para Residentes. España. Ed. biomédica Pfizer. 2006 Capitulo 21, p 217.

15. CASTELLANOS SÁNCHEZ, Nataly; MUÑOZ ORDOÑEZ, Milena. Tesis: escalas de valoración de riesgo: estrategia inicial y Prioritaria en la prevención de úlceras por presión. Bogotá. 2008. Pág.24.

16. Venegas Brenes, G.; Castro Céspedes, J.; Solano Madrigal, M. Programa para la prevención de úlceras por presión en personas adultas mayores. Rev. Enfermería Actual en Costa Rica.2010, No.18.

17. Flores MI. De la Cruz OS. Ortega VMC. Hernández MS. Cabrera PMF. Prevalencia de úlceras por presión atendidos en el Instituta Nacional de Cardiología Ignacio Chávez. Revista Mexicana de Enfermería Cardiología. Enero-Agosto 2010; 18(1-2): 13-17.

18. Vela-Anaya G, Magnitud del Evento Adverso. Úlceras por presión. Rev. Enferm Inst Mex Seguro Soc. 2013; 21(01): 3-8.

19. Soldevilla Agreda JJ. Torra i Bou JE. Epidemiología de las úlceras por presión en España. Estudio piloto en la comunidad autónoma de la Rioja, en Gerokomos. 1999; 10(2): 75-86.

20. Soldevilla Agreda JJ. Navarro Rodríguez S. Aspectos legales relacionados con las úlceras por presión. Gerokomos. 2006; 17(4), 203-224. 


\section{ANEXO I}

\section{DATOS DE IDENTIFICACION DEL PACIENTE}

Nombre:

Fecha:

No. Expediente:

Fecha ingreso:

Edad. Género: $F() M() \quad$ Fecha de valoración:

Servicio: $\mathrm{N}^{\circ}$ cama:

Diagnóstico:

Presenta UPP a su ingreso:

Grado de riesgo de UPP

Fecha de egreso:

Instrucciones: Registrar los datos referentes a la UPP

\begin{tabular}{|l|l|l|l|}
\hline $\begin{array}{l}\text { Sitio de } \\
\text { localiza- } \\
\text { ción }\end{array}$ & $\begin{array}{l}\text { Grado } \\
\text { UPP }\end{array}$ & $\begin{array}{l}\text { Tipo de } \\
\text { tejido }\end{array}$ & Fecha \\
\hline 1 & & & \\
\hline 2 & & & \\
\hline 3 & & & \\
\hline 4 & & & \\
\hline 5 & & & \\
\hline
\end{tabular}

Estadio I Piel integra. Eritema cutáneo.

Estadio II Úlcera superficial que tiene aspecto de abrasión, ampolla o cráter superficial.

Estadio III Pérdida total del grosor de la piel que implica lesión o necrosis del tejido subcutáneo.

Estadio IV Presenta lesiones con cavernas, tunelizaciones o trayectos sinuosos.

Tratamiento: 